\title{
ROBOTIC FORCE CONTROL BY USING ESTIMATED CONTACT FORCE
}

\author{
Han-Pang Huang \\ Wen-Liang Treng \\ Robotics Laboratory \\ Department of Mechanical Engineering \\ National Taiwan University \\ Taipei, Taiwan 10764 \\ Republic of China
}

\begin{abstract}
In general, the contact force, which is used in controller design for robotic compliant motion, can be either explicitly calculated through complicated computation or measured by expensive force sensors. In this paper, we propose a force control strategy by using the estimated contact force. The estimation of the contact force and states are primarily based on our previous observer design. It will be shown that the controller achieves asymptotic stability.
\end{abstract}

\section{INTRODUCTION}

Force control of robot systems has been studied by many researchers $[1,7,9,11]$. Among those approaches there are two main branches. One is feedback the contact force by using expensive force sensors [9]. The other is direct calculation of the contact force by using the constrained manipulator model $[2,6,8]$. In some cases it may be inadequate to measure the contact force. In other cases it may be either very costly to measure or very complicated to compute the contact force. Therefore, it is desired to design a force controller based on the estimated contact force.

The robot system under consideration is described by equations of the type [3]

$$
\begin{gathered}
M(q) \ddot{q}+F(q, \dot{q})=u+J^{T}(q) \lambda \\
\phi(q)=0 \\
y=C\left[\begin{array}{l}
\dot{q} \\
q
\end{array}\right]
\end{gathered}
$$

where $q \in R^{n}$ is the generalized displacement; $M(q)$ is an $n \times n$ inertial matrix function; $F(q, \dot{q})$ is an $n$ dimensional vector function, containing the Coriolis, the centrifugal and the gravitational terms; $u \in R^{n}$ is the generalized control input; $\phi(q)$ is the $\mathrm{m}$ dimensional constraint vector function; $J(q)=\frac{\partial \phi(q)}{\partial q}$ is an $m \times n$ Jacobian matrix; $\lambda \in R^{m}$ is the generalized contact force vector associated with the constraints; $y \in R^{p}$ is the output vector and $\mathrm{C}$ is a $p \times 2 n$ constant matrix. Our objective is to find a stabilizing control law for the system (1) (3), in the case where only $y(t)$ can be measured, but not necessarily $x(t)$ and $\lambda(t)$.

For a linear time-invariant differential system, it is well known [5] that a stabilizing control law can be constructed as long as the differential system is both stabilizable and detectable. However, the system under consideration is nonlinear as well as differential-and-algebraic type. These characteristics pose a lot of difficulty in designing a stabilizing control law for the system (1) (3). In our approach, the results from Vidyasagar [10] will be used and our previous observer design [3] will be used for the estimation of the contact force and states.

In order to facilitate the following development, the system (1) (3) is converted into the following form by using a nonlinear transformation [7]

$$
\begin{aligned}
E_{1} \bar{M}\left(x_{2}\right) E_{2}^{T} \bar{x}_{2}+E_{1} \bar{F}\left(x_{2}, \dot{x}_{2}\right) & =E_{1} T^{T}\left(x_{2}\right) u \\
& +E_{1} T^{T}\left(x_{2}\right) J^{T}\left(x_{2}\right) \lambda \\
E_{2} \bar{M}\left(x_{2}\right) E_{2}^{T} \bar{x}_{2}+E_{2} \bar{F}\left(x_{2}, \dot{x}_{2}\right) & =E_{2} T^{T}\left(x_{2}\right) u \\
x_{1} & =0
\end{aligned}
$$




$$
y=C\left[\begin{array}{c}
T(x) \dot{x} \\
Q(x)
\end{array}\right]=C\left[\begin{array}{c}
\frac{\partial \Omega\left(x_{2}\right)}{\partial x_{2}} \dot{x}_{2} \\
\dot{x}_{2} \\
\Omega\left(x_{2}\right) \\
x_{2}
\end{array}\right]
$$

where

$$
\begin{aligned}
\bar{M}\left(x_{2}\right) & =T^{T}\left(x_{2}\right) M\left(Q\left(x_{2}\right)\right) T\left(x_{2}\right) \\
\bar{F}\left(x_{2}, \dot{x}_{2}\right) & =T^{T}\left(x_{2}\right)\left[F\left(Q\left(x_{2}\right), T\left(x_{2}\right) \dot{x}_{2}\right)\right. \\
& \left.+M\left(Q\left(x_{2}\right)\right) \dot{T}\left(x_{2}\right) \dot{x}_{2}\right]
\end{aligned}
$$

Our problem turns out to design the observer and controller for the transformed system (4)-(7). Throughout this paper, we assume that the system is always observable (or detectable) and that the subsystem (5) is always controllable (or stabilizable). The detailed discussion of observability and controllability for constrained robot systems are discussed in our other paper [4].

The paper is organized as follows. In section II, we present some preliminary results, including our previous observer design and Vidyasagar's results. In section III, we present the main theorem concerning asymptotic stability. Section IV gives an illustrative example and Section V contains the conclusion.

\section{PRELIMINARIES}

In this section, we briefly summarize our previous observer design [3] and some results from Vidyasagar [10].

First, the observer design is summarized. Given the following assumption

\section{Assumption 1}

(1) $\left[\begin{array}{c}{\left[E_{2} \bar{M}\left(x_{2}\right) E_{2}^{T}\right]^{-1} E_{2} T^{T}\left(x_{2}\right)} \\ 0\end{array}\right] u=P^{-1} \bar{C}^{T} \sum_{i=1}^{n} R_{i}\left(x_{2}\right) u_{i}$

(2) $\nabla R_{i}\left(x_{2}\right)=L_{i}\left(x_{2}\right) \bar{C}, \quad\left\|L_{i}\left(x_{2}\right)\right\| \leq H_{i} \quad i=1, \cdots, n$ where $P^{-1}=\left[\begin{array}{l}P_{1} \\ P_{2}\end{array}\right], P_{1} \in R^{(n-m) \times 2(n-m)}, P_{2} \in R^{(n-m) \times}$ $P$ is a $2(n-m) \times 2(n-m)$ positive matrix; $H_{i}$ is a positive number; and $P_{2} \bar{C}^{T} \bar{C}=0$.

Then the observer structure is chosen as follows

$$
\dot{\hat{x}_{2}}=\hat{\dot{x}}_{2}+G_{1}^{\circ} \bar{C}\left[\begin{array}{l}
\dot{x}_{2}-\hat{\dot{x}}_{2} \\
x_{2}-\hat{x}_{2}
\end{array}\right]
$$

$$
\begin{aligned}
& E_{1} \bar{M}\left(\hat{x}_{2}\right) E_{2}^{T} \dot{\hat{\dot{x}}}_{2}=-E_{1} \bar{F}\left(\hat{x}_{2}, \hat{\dot{x}}_{2}\right)+E_{1} T^{T}\left(\hat{x}_{2}\right) J^{T}\left(\hat{x}_{2}\right) \hat{\lambda} \\
&+E_{1} \bar{M}\left(\hat{x}_{2}\right) E_{2}^{T} G_{3}^{o} \bar{C}\left[\begin{array}{l}
\dot{x}_{2}-\hat{\dot{x}}_{2} \\
x_{2}-\hat{x}_{2}
\end{array}\right]+E_{1} T^{T}\left(\hat{x}_{2}\right) u \\
& E_{2} \bar{M}\left(\hat{x}_{2}\right) E_{2}^{T} \dot{\hat{\dot{x}}}_{2}=-E_{2} \bar{F}\left(\hat{x}_{2}, \hat{\dot{x}}_{2}\right)+E_{2} \bar{M}\left(\hat{x}_{2}\right) E_{2}^{T} G_{2}^{o} \bar{C} \\
& \\
& \quad\left[\begin{array}{l}
\dot{x}_{2}-\hat{\dot{x}}_{2} \\
x_{2}-\hat{x}_{2}
\end{array}\right]+E_{2} \bar{M}\left(\hat{x}_{2}\right) E_{2}^{T} P^{-1} \bar{C}^{T} \bar{C} . \\
& {\left[\begin{array}{l}
\dot{x}_{2}-\hat{\dot{x}}_{2} \\
x_{2}-\hat{x}_{2}
\end{array}\right] \sum_{i=1}^{n} H_{i}\left\|u_{i}\right\|+E_{2} T^{T}\left(\hat{x}_{2}\right) u }
\end{aligned}
$$

where $G_{1}^{o}, G_{2}^{o}$, and $G_{3}^{o}$ are constant matrices.

We have the following results.

Theorem 1: [3] Consider the system (4)(5)(7). If the following conditions are satisfied

(1) Assumption 1 holds,

(2) $P \nabla \tilde{F}\left(x_{2}, \dot{x}_{2}\right)-P\left[\begin{array}{l}G_{2}^{o} \bar{C} \\ G_{1}^{o} \bar{C}\end{array}\right] \nabla\left[\begin{array}{l}\dot{x}_{2} \\ x_{2}\end{array}\right]$ is uniformly negative definite for all $x_{2}, \dot{x}_{2} \in R^{n-m}$ and some $\epsilon>0$; where

$$
\tilde{F}\left(x_{2}, \dot{x}_{2}\right)=\left[\begin{array}{c}
-\left(E_{2} \bar{M}\left(x_{2}\right) E_{2}^{T}\right)^{-1} E_{2} \bar{F}\left(x_{2}, \dot{x}_{2}\right) \\
\dot{x}_{2}
\end{array}\right]
$$

and

$$
\nabla \tilde{F}=\left[\frac{\partial \tilde{F}}{\partial \dot{x}_{2}}, \frac{\partial \tilde{F}}{\partial x_{2}}\right]
$$

(3) $E_{1} T^{T}\left(x_{2}\right) J^{T}\left(x_{2}\right)$ and $E_{1} T^{T}\left(\hat{x}_{2}\right) J^{T}\left(\hat{x}_{2}\right)$ are nonsingular for all $x_{2}, \hat{x}_{2} \in R^{n-m}$,

then the observer defined by Eqns.(10)-(12) is an asymptotic observer in the sense that

$$
e^{T}(t) P e(t) \leq e^{T}\left(t_{0}\right) P e\left(t_{0}\right) e^{-\eta\left(t-t_{0}\right)}, \forall t \geq t_{0}
$$

where

$$
e(t)=\left[\begin{array}{l}
\dot{x}_{2}(t)-\hat{\dot{x}}_{2} \\
x_{2}(t)-\hat{x}_{2}
\end{array}\right], \quad \eta=\frac{2 \epsilon e^{T} e}{e^{T} P e}
$$

In addition, the estimated contact force $\hat{\lambda}$ converges to $\lambda$.

Note that the complete information of the original states can be obtained by the following transformation

$$
\hat{q}=\left[\begin{array}{l}
\hat{q}_{1} \\
\hat{q}_{2}
\end{array}\right]=Q(\hat{x})=\left[\begin{array}{c}
\Omega\left(\hat{x}_{2}\right) \\
\hat{x}_{2}
\end{array}\right]
$$

and

$$
\hat{\dot{q}}=\left[\begin{array}{c}
\hat{\dot{q}_{1}} \\
\dot{\dot{q}_{2}}
\end{array}\right]=T(\hat{x}) \hat{\dot{x}}=\left[\begin{array}{cc}
I_{m} & \frac{\partial \Omega\left(x_{2}\right)}{\partial x_{2}} \\
0 & I_{n-m}
\end{array}\right]_{x_{2}=\dot{x}_{2}}\left[\begin{array}{c}
0 \\
\hat{\dot{x}_{2}}
\end{array}\right]
$$

Next, some results from Vidyasagar [10] are summarized. Consider the nonlinear system

$$
\begin{aligned}
& \dot{x}(t)=f(t, x(t), u(t)) \\
& y(t)=r(t, x(t))
\end{aligned}
$$

Given the following assumption 


\section{Assumption 2}

- $f$ is continuously differentiable and vanishes when all of its arguments except $t$ vanish; further, there are constants $\alpha$ and $c$ such that

$$
\left\|\nabla_{x} f(t, x, u)\right\| \leq \alpha \quad \forall t \geq 0 \forall x \in B_{c} \forall u \in B_{c}
$$

- $r$ is continuous and $r(0,0)=0$.

Then we have the following result

Theorem 2 [10] Suppose the system (17)(18) satisfies Assumption 2 and is stabilizable and detectable. If the system is stabilized by the control law

$$
u(t)=h(t, x(t))
$$

then it is also stabilized by the control law

$$
u(t)=h(t, z(t))
$$

where $z(t)$ is the output of a detector for $x(t)$.

Theorem 2 is remarkable in that the observer and the feedback controller for a nonlinear system can be designed separately if certain assumptions are satisfied. This result will be used in the next section for controller design.

\section{STABILIZING CONTROL LAW}

A constrained robot system is a highly nonlinear dynamic system. Particularly, it consists of differential equations and algebraic equations. It is quite difficult to design both observer and feedback controller simultaneously. Fortunately, Theorem 2 given ia: Section II provides a way to separately design the observer and the controller. In our design, the observer will have the same form as in Section II and the controller will be computed-torque like. We will show that the combination of the observer and the controller gives a stabilizing control law.

Suppose we select a computed torque control law as follows [7].

$$
\begin{aligned}
T^{T}\left(x_{2}\right) u & =\bar{M}\left(x_{2}\right) E_{2}^{T} \bar{x}_{2 d}+\bar{F}\left(x_{2}, \dot{x}_{2}\right)-T^{T}\left(x_{2}\right) J^{T}\left(x_{2}\right) \lambda_{d} \\
& +\bar{M}\left(x_{2}\right) E_{2}^{T} G_{v}\left(\dot{x}_{2 d}-\dot{x}_{2}\right)+\bar{M}\left(x_{2}\right) E_{2}^{T} G_{d}\left(x_{2 d}-x_{2}\right) \\
& +E_{1}^{T} G_{j} E_{1} T^{T}\left(x_{2}\right) J^{T}\left(x_{2}\right)\left(\lambda-\lambda_{d}\right)
\end{aligned}
$$

where $G_{v}, G_{d} \in R^{(n-m) \times(n-m)}, G_{f} \in R^{m \times m}$ are constant matrices; $x_{2 d}=E_{2} X\left(q_{d}\right), q_{d}$ are desired displacement vector; $\lambda_{d}$ is desired contact force vector. Substituting (21) into
Eqns.(4),(5), it is easy to verify that if the following conditions are satisfied, then the system is stable:

Condition 1

(1) $G_{v}, G_{d}$ are symmetric and positive definite.

(2) $G_{f}$ is symmetric and nonnegative definite.

(3) $E_{1} T^{T}\left(x_{2}\right) J^{T}\left(x_{2}\right)$ is nonsingular for all $x_{2} \in R^{n-m}$.

If we introduce the control law which has the same structure as Eq.(21) but feedbacks the estimated states $\hat{x}_{2}, \dot{\hat{x}}_{2}$ and $\hat{\lambda}$ in stead of the real states $x_{2}, \dot{x}_{2}$ and $\lambda$, then the control law can be represented by

$$
\begin{aligned}
T^{T}\left(\hat{x}_{2}\right) u & =\bar{M}\left(\hat{x}_{2}\right) E_{2}^{T} \bar{x}_{2 d}+\bar{F}\left(\hat{x}_{2}, \hat{\dot{x}}_{2}\right)-T^{T}\left(\hat{x}_{2}\right) J^{T}\left(\hat{x}_{2}\right) \lambda_{d} \\
& +\bar{M}\left(\hat{x}_{2}\right) E_{2}^{T} G_{v}\left(\dot{x}_{2 d}-\dot{x}_{2}\right)+\bar{M}\left(\hat{x}_{2}\right) E_{2}^{T} G_{d}\left(x_{2 d}-\hat{x}_{2}\right) \\
& +E_{1} G_{f} E_{1} T^{T}\left(\hat{x}_{2}\right) J^{T}\left(\hat{x}_{2}\right)\left(\hat{\lambda}-\lambda_{d}\right)
\end{aligned}
$$

We have the following results:

Theorem 3: Consider the dynamic system defined by Eqns. $(4)(5)(7)$. Suppose we select the observer structure as (10)(12) and the control law as (22), if the following conditions are satisfied:

(1) There exists a real $\rho>0$, such that for all $\left\|\left[\begin{array}{l}\dot{x}_{2}-\dot{x}_{2 d} \\ \dot{x}_{2}-x_{2 d}\end{array}\right]\right\|$ $<\rho, \bar{f}\left(\dot{x}_{2}, x_{2}, u\right)$ is continuously differentiable with respect to $\dot{x}_{2}, x_{2}, u$, and $h\left(x_{2}, \dot{x}_{1}, x_{2 d}, \dot{x}_{2 d}\right)$ is continuously differentiable with respect to $x_{2}, \dot{x}_{2}$.

(2) For all $\left\|\left[\begin{array}{c}\dot{x}_{2}-\dot{x}_{2 d} \\ x_{2}-x_{2 d}\end{array}\right]\right\|<\rho,\left\|\nabla_{z} \bar{f}\left(\dot{x}_{2}, x_{2}, u\right)\right\|$ and $\|$ $\nabla_{x} h\left(x_{2}, \dot{x}_{2}, x_{2 d}, \dot{x}_{2 d}\right) \|$ are uniformly bounded by $\theta_{1}$ and $\theta_{2}$, respectively.

(3) All conditions of Theorem 1 are satisfied.

(4) The controller gains $G_{v}, G_{d}, G_{f}$ are selected as the Condition 1.

Note that

$$
\left[\begin{array}{l}
\dot{x}_{2 d} \\
x_{2 d}
\end{array}\right] \triangleq P_{d}\left(\dot{x}_{2 d}, x_{2 d}\right)=\tilde{F}\left(x_{2 d}, \dot{x}_{2 d}\right)
$$

and the matrix functions $\bar{f}$ and $\mathrm{h}$ are defined by

$$
\begin{gathered}
\tilde{f}\left(\dot{x}_{2}, x_{2}, u\right)=\tilde{F}\left(x_{2}, \dot{x}_{2}\right)+ \\
{\left[\begin{array}{c}
\left(E_{2} \bar{M}\left(x_{2}\right) E_{2}^{T}\right)^{-1} E_{2} T^{T}\left(x_{2}\right) \\
0
\end{array}\right] u} \\
\left(\hat{x}_{2}, \hat{\dot{x}}_{2}, x_{2 d}, \dot{x}_{2 d}\right)=u
\end{gathered}
$$

$\mathrm{u}$ is selected as Eq.(22).

then for $\left\|X\left(t_{0}\right)-X_{d}\left(t_{0}\right)\right\|<\delta$, the system states $x_{2}, \dot{x}_{2}$ and 
$\lambda$ can track the desired states $x_{2 d}, \dot{x}_{2 d}$ and $\lambda_{d}$, respectively. Here $X=\left[\begin{array}{c}\dot{x}_{2} \\ x_{2}\end{array}\right], \quad X_{d}=\left[\begin{array}{c}\dot{x}_{2 d} \\ x_{2 d}\end{array}\right]$.

Proof: Since the controller gains are selected to satisfy the Condition 1 , then according to [10], there exist a continuously differentiable scalar function $V_{1}$, and functions $\phi_{1}, \phi_{2}, \phi_{3}$ belonged to $K[12]$, such that

$$
\begin{gathered}
\phi_{1}\left(\left\|e_{1}\right\|\right) \leq V_{1}\left(e_{1}\right) \leq \phi_{2}\left(\left\|e_{1}\right\|\right) \\
\dot{V}_{1}\left(e_{1}\right)=\nabla_{e_{1}} V_{1}\left(e_{1}\right)\left(f\left(X, h\left(X, X_{d}\right)\right)-g\left(X_{d}\right)\right) \\
\leq-\phi_{3}\left(\left\|e_{1}\right\|\right) \\
\left\|\nabla_{e_{1}} V_{1}\left(e_{1}\right)\right\| \leq L
\end{gathered}
$$

for all $t \geq 0$ and for all $\left\|e_{1}\right\| \leq \rho$; where $L$ is a positive constant, $\nabla_{e_{1}} V_{1}\left(e_{1}\right)$ is the gradient of $V_{1}$ with respect to $e_{1}$; $g\left(X_{d}\right)=\dot{x}_{d}$ and $e_{1}=X-X_{d}$.

Since condition (2) holds, there exist $\theta_{1}>0, \theta_{2}>0$ such that for all $t \geq t_{0}$, all $\left\|e_{1}\right\|<\rho,\left\|e_{2}\right\|<\rho,\|u\|<\rho$ and $\|v\|<\rho$ the following equations hold

$$
\begin{aligned}
& \left\|h\left(X, X_{d}\right)-h\left(\hat{X}, X_{d}\right)\right\| \leq \theta_{1}\|X-\hat{X}\| \leq \theta_{1}\left\|e_{1}-e_{2}\right\| \\
& \qquad\|\bar{f}(X, u)-\bar{f}(X, v)\| \leq \theta_{2}\|u-v\| \\
& \text { where } e_{2}=\hat{X}-X_{d}, \quad \hat{X}=\left[\begin{array}{l}
\hat{\dot{x}}_{2} \\
\hat{x}_{2}
\end{array}\right] .
\end{aligned}
$$

Because all conditions of Theorem 1 hold, the observer is an asymptotic observer, according to [10] there exist a Lyapunov function $V_{2}$, which is function of $\left(e_{1}-e_{2}\right)$ or expressed as $V_{2}\left(e_{1}-e_{2}\right)$, and functions $\psi_{1}, \psi_{2}, \psi_{3}$ belonged to $\mathrm{K}$ such that

$$
\begin{gathered}
\psi_{1}\left(\left\|e_{1}-e_{2}\right\|\right) \leq V_{2}\left(e_{1}-e_{2}\right) \leq \psi_{2}\left(\left\|e_{1}-e_{2}\right\|\right) \\
\dot{V}_{2}\left(e_{1}-e_{2}\right)=\nabla_{\left(e_{1}-e_{2}\right)} V_{2}\left(e_{1}-e_{2}\right)\left[\bar{f}(X, u)-g\left(X_{d}\right)\right] \\
\leq-\psi_{3}\left(\left\|e_{1}-e_{2}\right\|\right)
\end{gathered}
$$

From (21)(26) it can be shown that there exists $\delta_{1}>0$ such that

$$
\left\|h\left(\hat{X}, X_{d}\right)\right\|<\rho
$$

for $\left\|e_{2}\right\|<\delta_{1}$ and $t \geq t_{0}$. Let $\epsilon_{1}=\min \left\{\frac{\epsilon}{2}, \frac{\delta_{1}}{2}\right\}$. Select $\epsilon_{2}$ for $\phi_{2}\left(\epsilon_{2}\right)<\phi_{1}\left(\epsilon_{1}\right)$, and $\delta_{2}$ for $\delta_{2} \leq \epsilon_{2}$ and $L \theta_{1} \theta_{2} \delta_{2}<\phi_{3}\left(\epsilon_{2}\right)$, and $\delta_{3}$ for $\psi_{2}\left(\delta_{3}\right)<\psi_{1}\left(\delta_{2}\right)$. If we choose

$$
\delta=\min \left(\rho, \frac{\delta_{3}}{2}\right)
$$

then

$$
\delta \leq \delta_{3} / 2<\delta_{2}<\epsilon_{2}<\epsilon_{1} \leq \delta_{1} / 2
$$

Suppose $\left\|e_{1}\left(t_{0}\right)\right\|=\left\|X\left(t_{0}\right)-X_{d}\left(t_{0}\right)\right\|<\delta$ and $\| e_{1}\left(t_{0}\right)-$ $e_{2}\left(t_{0}\right) \|<\delta$. Since the stability of the estimated error state vector is independent of the choice of the control law, Eqns. (28)(29) always hold.

Re-write (25) in terms of the control $u$, we have

$$
\dot{V}_{1}\left(e_{1}\right)=\nabla_{e_{1}} V_{1}\left(e_{1}\right)\left[\bar{f}(X, u)-g\left(X, X_{d}\right)\right]
$$

If we introduce the feedback control in the form of Eq.(22), then (23) becomes

$$
\begin{aligned}
\dot{V}_{1}\left(e_{1}\right)= & \nabla_{e_{1}} V_{1}\left(e_{1}\right)\left[\bar{f}\left(X, h\left(\hat{X}, X_{d}\right)\right)-g\left(X_{d}\right)\right] \\
= & \nabla_{e_{1}} V_{1}\left(e_{1}\right)\left[\bar{f}\left(X, h\left(X, X_{d}\right)\right)-g\left(X_{d}\right)\right] \\
& +\nabla_{e_{1}} V_{1}\left(e_{1}\right)\left[\bar{f}\left(X, h\left(\hat{X}, X_{d}\right)\right)-\bar{f}\left(X, h\left(X, X_{d}\right)\right)\right] \\
\leq & -\phi_{3}\left(\left\|e_{1}\right\|\right)+L \theta_{1} \theta_{2} \delta_{2} \\
\leq & -\phi_{3}\left(\epsilon_{2}\right)+L \theta_{1} \theta_{2} \delta_{2}<0
\end{aligned}
$$

From Eqns. (24)(34), we have

$$
\begin{gathered}
\phi_{1}\left(\left\|e_{1}\right\|\right) \leq V_{1}\left(e_{1}\right) \leq \phi_{2}\left(\left\|e_{1}\right\|\right) \\
\dot{V}_{1}\left(e_{1}\right)=\nabla_{e_{1}} V_{1}\left(e_{1}\right)\left(\bar{f}\left(x, h\left(\hat{X}, X_{d}\right)\right)-g\left(X_{d}\right)\right)<0
\end{gathered}
$$

for all $t \geq t_{0},\left\|e_{1}\right\|<\delta$. Thus, for $\left\|e_{1}\left(t_{0}\right)\right\|<\delta$ and $\left\|e_{1}\left(t_{0}\right)-e_{2}\left(t_{0}\right)\right\|<\delta$, the tracking error state vector $e_{1}(t)$ will converge to zero as time approaching infinity. Namely, the system states $x_{2}, \dot{x}_{2}$ can track the desired states $x_{2 d}, \dot{x}_{2 d}$.

Consider Eqns. (4)(11), the closed loop system is given as

$$
\begin{aligned}
E_{1} \bar{M}\left(x_{2}\right) E_{2}^{T} \ddot{x}_{2} & +E_{1} \bar{F}\left(x_{2}, \dot{x}_{2}\right)=E_{1} T^{T}\left(x_{2}\right) T^{-T}\left(\hat{x}_{2}\right) \\
& \left\{\bar{M}\left(\hat{x}_{2}\right) E_{2}^{T} \ddot{x}_{2 d}+\bar{F}\left(\hat{x}_{2}, \hat{\dot{x}}_{2}\right)\right. \\
& -T^{T}\left(\hat{x}_{2}\right) J^{T}\left(\hat{x}_{2}\right) \lambda_{d}+\bar{M}\left(\hat{x}_{2}\right) E_{2}^{T} G_{v} \\
& \left(\dot{x}_{2 d}-\hat{\dot{x}}_{2}\right)+\bar{M}\left(\hat{x}_{2}\right) E_{2}^{T} G_{d}\left(x_{2 d}-\hat{x}_{2}\right) \\
& \left.+E_{1}^{T} G_{f} E_{1}^{T} T\left(\hat{x}_{2}\right) J^{T}\left(\hat{x}_{2}\right)\left(\hat{\lambda}-\lambda_{d}\right)\right\} \\
& +E_{1} T^{T}\left(x_{2}\right) J^{T}\left(x_{2}\right) \lambda
\end{aligned}
$$

Since $\hat{x}_{2} \rightarrow x_{2}, x_{2} \rightarrow x_{2 d}, \hat{\dot{x}}_{2} \rightarrow \dot{x}_{2}, \dot{x}_{2} \rightarrow \dot{x}_{2 d}, \hat{\lambda} \rightarrow \lambda$ as $t \rightarrow \infty$, Eq.(37) can be simplified as 


$$
\left(I+G_{f}\right) E_{1} T^{T}\left(x_{2}\right) J^{T}\left(x_{2}\right)\left(\lambda-\lambda_{d}\right) \rightarrow 0
$$

as $t \rightarrow \infty$. Since $E_{1} T^{T}\left(x_{2}\right) J^{T}\left(x_{2}\right)$ is nonsingular and $G_{f}$ is nonnegative definite, the contact force vector $\lambda$ will also converge to the desired contact force vector $\lambda_{d}$.

Q.E.D.

\section{AN EXAMPLE}

In this section, an example is given to illustrate the above development. Suppose a constrained dynamic system is given by

$$
\begin{gathered}
\ddot{q}_{1}=u_{1} \\
\left(100-4 \dot{q}_{2}^{2}\right) \dot{q}_{2}+\dot{q}_{2}^{3}+100 q_{2}-4 q_{2} \dot{q}_{2}^{2}=u_{2}
\end{gathered}
$$

The constraint and output equations are

$$
\begin{gathered}
\phi\left(q_{1}, q_{2}\right)=q_{1}^{2}-q_{2} \\
y=\left[\begin{array}{l}
q_{1}+q_{2} \\
q_{2}+\dot{q}_{2}
\end{array}\right]
\end{gathered}
$$

where $q_{1}, q_{2}, u, u_{2} \in R$ and $y \in R^{2 \times 1}$

By introducing the Lagrange multiplier and applying the McClamroch and Wang's nonlinear transformation, Eqns.(39) (42) can be transformed into

$$
\begin{gathered}
2 \dot{x}_{2}^{2}+2 x_{2} \bar{x}_{2}=u_{1}+\lambda \\
\bar{x}_{2}=-0.01 \dot{x}_{2}^{3}-x_{2}+0.02 x_{2} u_{1}+0.01 u_{2} \\
x_{1}=0 \\
y=\left[\begin{array}{lll}
1 & 1 & 0 \\
0 & 1 & 1
\end{array}\right]\left[\begin{array}{l}
x_{2}^{2} \\
x_{2} \\
\dot{x}_{2}
\end{array}\right]
\end{gathered}
$$

By singular value decomposition, a linear output can be constructed from (46) as

$$
\bar{y}=\left[\begin{array}{ll}
0 & 2
\end{array}\right] y=2 x_{2}+2 \dot{x}_{2}
$$

Select the observer structure as follows

$$
\begin{aligned}
& 2 \dot{\hat{\dot{x}}}_{2}+2 \hat{x}_{2} \dot{\dot{\dot{x}}}_{2}=u_{1}+\hat{\lambda}+2 \hat{x}_{2} G_{3}^{o}\left(x_{2}+\dot{x}_{2}-\hat{x}_{2}-\hat{\dot{x}}_{2}\right) \\
& \dot{\hat{\dot{x}}}_{2}=-0.01 \hat{\dot{x}}_{2}^{o}-\hat{x}_{2}+0.02 \hat{x}_{2} u_{1}+0.01 u_{2}+2 G_{2}^{o} \\
& \left(x_{2}+\dot{x}_{2}-\hat{x}_{2}-\hat{\dot{x}}_{2}\right)+4 H\left(x_{2}+\dot{x}_{2}-\hat{x}_{2}-\hat{\dot{x}}_{2}\right)\left\|u_{1}\right\|(49) \\
& \dot{\hat{x}}_{2}=\hat{\dot{x}}_{2}+2 G_{1}^{o}\left(x_{2}+\dot{x}_{2}-\hat{x}_{2}-\hat{\dot{x}}_{2}\right)
\end{aligned}
$$

Select $H=0.15$ and the observer gains as $G_{1}^{\circ}=-7, G_{2}^{o}=5.5$,
$G_{3}^{o}=0.05$, then according to Theorem 1 , the observer defined by Eqns. (47)-(50) is an asymptotic observer for the system defined by Eqns. (43)(44)(47).

A computed-torque controller is designed as

$$
\begin{aligned}
u_{1}= & G_{f}\left(\hat{\lambda}-\lambda_{d}\right)-\lambda_{d}+2 \hat{x}_{2} \bar{x}_{2 d}+2 \hat{x}_{2} G_{v}\left(\dot{x}_{2 d}-\hat{\dot{x}}_{2}\right) \\
& +2 \hat{\dot{x}}_{2} G_{d}\left(x_{2 d}-\hat{x}_{2}\right)+2 \hat{\dot{x}}_{2}^{2} \\
u_{2}= & -2 \hat{x}_{2} u_{1}+100\left(\bar{x}_{2 d}+G_{v}\left(\dot{x}_{2 d}-\hat{\dot{x}}_{2}\right)+G_{d}\left(x_{2 d}-\hat{x}_{2}\right)\right. \\
& \left.+0.001 \hat{\dot{x}}_{2}^{3}+\hat{x}_{2}\right)
\end{aligned}
$$

The controller gains are chosen as $G_{f}=1, G_{v}=7$, and $G_{d}=$ 12. Suppose the initial conditions are

$$
x_{2}(0)=1, \dot{x}_{2}(0)=1, \hat{x}_{2}(0)=0.5, \hat{\dot{x}}_{2}(0)=0.5
$$

$$
\lambda(0)=4, \hat{\lambda}(0)=3
$$

and the desired values are

$$
x_{2 d}=t, \dot{x}_{2 d}=1, \lambda_{d}=10,
$$

then the results are shown in Fig. (1) to Fig. (3).

From Fig.(1)-(3), we know that the estimated states $\hat{x}_{2}, \hat{\dot{x}}_{2}$ and $\hat{\lambda}$ will converge to the system states $x_{2}, \dot{x}_{2}$ and $\lambda$, respectively. Moreover, the controller design based on estimated states and estimated contact force also show the capability of tracking the desired input.

\section{CONCLUSION}

In this paper, we have shown that the constrained robot system can be stabilized by a controller based on estimated contact force and states. The tool used in obtaining this result is the results given by Vidyasagar. Although the controller used here is the form of computed-torque, it can be arbitrary form as long as the relevant assumptions are met. The above idea is also demonstrated in an example. Since the contact force can be estimated and the controller based on this estimated force results in a stabilizing control law, we feel that this result should be useful for robot force control. 


\section{REFERENCE}

[1] Huang, H.P., N.H. McClamroch, "Time-Optimal Control for a Contour Following Problem." IEEE J. of Robotics and Automation, Vol.4, No.2, 140-149, April 1988.

[2] Huang, H.P., "The Unified Formulation of Constrained Robot Systems," Proc. of IEEE Conf. on Robotics and Automation, Philadelphia, 1988.

[3] Huang, H.P., Wen-Liang Tzeng, "An Observer Design for Constrained Robot Systems," Proc. of IEEE Conf. on Decision and Control, Austin, U.S.A., December 1988.

[4] Huang, H.P., Wen-Liang Tzeng, "Controllability and Observability of Constrained Systems," Proc. of the 12th National Symposium on Automatic Control, pp. 460 466, Hsin-Chu, Taiwan, R.O.C., Dec. 1988.

[5] Kailath, T., Linear Systems. Englewood Cliffs, N.J.: Prentice-Hall, 1980.

[6] McClamroch, N.H., H.P. Huang, "Dynamics of a Closed Chain Manipulator," Proc. of American Control Conference, Boston, 1985.

[7] McClamroch, N.H., D. Wang, "Feedback Stabilization and Tracking of Constrained Robots," Proc. of American Control Conference, 1987.

[8] McClamroch, N.H., "Singular Systems of Differential Equations as Dynamic Models for Constrained Robot Systems," Proc. of IEEE Conf. on Robotics and Automation, San Francisco, 1986.

[9] Raibert, M.H., J.J. Craig, "Hybrid Position/Force Control of Manipulators," ASME J. of Dynamic Systems, Measurement and Control, Vol. 102, pp. 126-133, June 1981.

[10] Vidyasagar, M., "On the Stabilization of Nonlinear Systems Using state Detection." IEEE Trans. on Automatic Control, Vol.25, No.3, 504-509, July 1980.

[11] Whitney, D.E., "Historical Perspective and State of the Art in Robot Force Control," The International Journal of Robotics Research, Vol. 6, No. 1, Spring 1987.

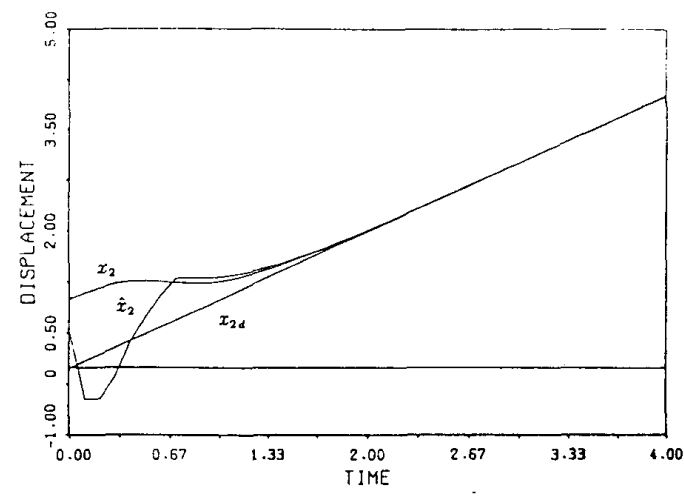

Fig.1 Displacement $x_{2}, \hat{x}_{2}$ and $x_{2 d}$

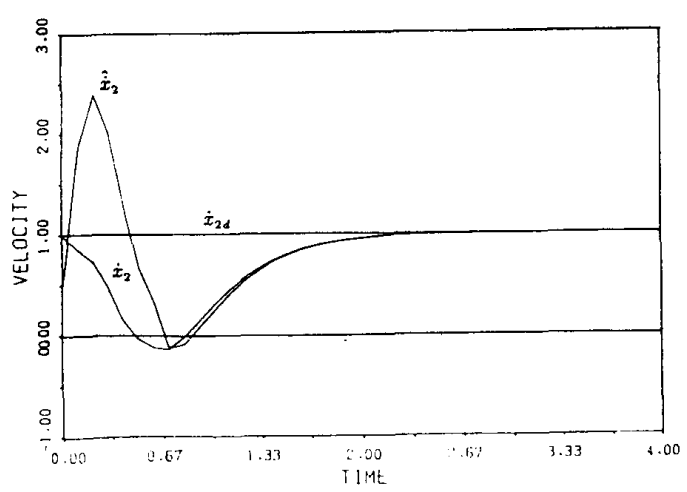

Fig. 2 Velocity $\dot{x}_{2}, \hat{\dot{x}}_{2}$ and $\dot{x}_{2 d}$

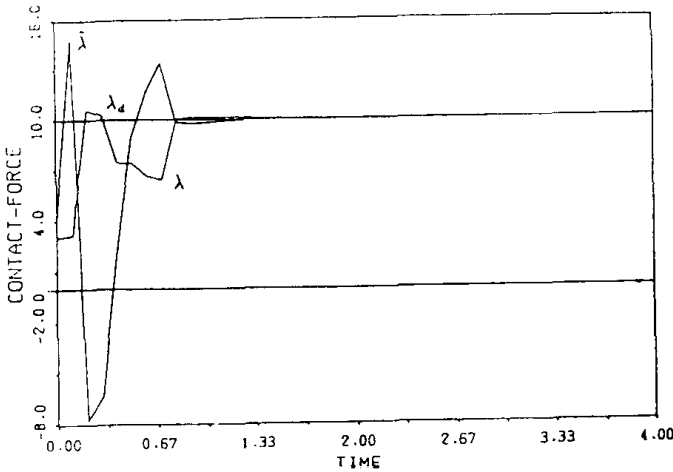

Fig.3 Contact Force $\lambda, \hat{\lambda}$ and $\lambda_{d}$ 